\title{
Lipoxygenase Contributes to the Oxidation of Lipids in Human Atherosclerotic Plaques
}

\author{
Virginia A. Folcik, ${ }^{\star}$ Rafael A. Nivar-Aristy, ${ }^{\mathbf{6}}$ Leonard P. Krajewski, ${ }^{\ddagger}$ and Martha K. Cathcart ${ }^{\star}$ \\ Departments of ${ }^{*}$ Cell Biology and ${ }^{\ddagger}$ Vascular Surgery, Cleveland Clinic Foundation, Cleveland, Ohio 44195; \\ and ${ }^{8}$ Department of Pathology, Ohio Permanente Medical Group, Parma, Ohio 44130
}

\begin{abstract}
Oxidized LDL is present in human atherosclerotic lesions, but the mechanisms responsible for oxidation in vivo have not been definitively demonstrated. Circumstantial evidence has implicated the enzyme 15-lipoxygenase as a contributor to the formation of oxidized lipids in this disease. To assess whether oxidized lipids are indeed formed by the action of 15-lipoxygenase on polyunsaturated fatty acids (PUFAs) in vivo, we have used a sensitive and specific method (chiral phase HPLC) to analyze the lipid oxidation products present in human atherosclerotic lesions. Human 15-lipoxygenase is an $\omega-6$ lipoxygenase that has previously been shown to oxidize esterified PUFA in a stereospecific manner, forming predominantly cholesteryl hydroperoxy-octadecadienoate $(13(S)$-HPODE) from cholesteryl linoleate substrate in LDL. This property allows its activity to be distinguished from nonenzymatic oxidation, which results in the formation of equal quantities of the $S$ and $R$ stereoisomers of the same oxidation product. A total of 80 specimens of human atherosclerotic plaque were analyzed. Esterified, oxidized linoleate was purified from human atherosclerotic lesions and from LDL oxidized by copper, and the chirality of these oxidation products was compared. There was significantly greater stereospecificity of oxidation in the oxidized linoleate from human atherosclerotic lesions. Even greater stereospecificity was detected in the HPODE derived from cholesteryl ester, purified from human lesions. Cholesteryl HPODE is the primary oxidation product from cholesteryl linoleate, the major esterified PUFA that accumulates in atherosclerotic vessels. Cholesteryl HPODE and its reduced form, cholesteryl hydroxy-octadecadienoate, were detected in all lesions analyzed. Neither the stereospecificity of oxidation nor the percentage of available substrate oxidized to primary oxidation products was correlated with the stage of disease of the lesions examined. We conclude that 15lipoxygenase contributes to the formation of oxidized lipids in human atherosclerotic lesions. (J. Clin. Invest. 1995. 96:504-510.) Key words: atherosclerosis • cholesteryl esters - lipid peroxidation • lipoproteins $\bullet$ lipoxygenases
\end{abstract}

Address correspondence to Martha K. Cathcart, Ph.D., Department of Cell Biology, Building NN1, Research Institute, Cleveland Clinic Foundation, 9500 Euclid Avenue, Cleveland, OH 44195. Phone: 216-4445222. FAX: 216-444-9404.

Received for publication 23 December 1994 and accepted in revised form 7 March 1995.

J. Clin. Invest.

(C) The American Society for Clinical Investigation, Inc.

0021-9738/95/07/0504/07 \$2.00

Volume 96, July 1995, 504-510

\section{Introduction}

In the last decade strong evidence has accumulated supporting the concept that oxidative processes, acting on the lipids and proteins of LDL in the vessel wall, participate in the progression of atherosclerotic disease (1-4). Evidence from in situ studies has demonstrated that oxidized LDL, macrophages, and 15lipoxygenase colocalize within human atherosclerotic lesions (5). We therefore chose to determine whether 15-lipoxygenase was acting upon the available lipid substrate within the lesions to cause its oxidation in vivo.

Early studies by Hamberg and Samuelsson (6) demonstrated that the oxidation of polyunsaturated fatty acids PUFAs ${ }^{1}$ by soybean 15 -lipoxygenase was stereospecific. Kuhn et al. (7) later developed a convenient chiral phase HPLC method and used it to analyze the PUFA oxidation products from a variety of lipoxygenase enzymes, confirming that stereospecificity was a fingerprint of lipoxygenase activity. These investigators found that mammalian lipoxygenase enzymes also specifically produce the $S$ enantiomer at the target positions of oxidation on their PUFA substrates (7). In contrast, nonenzymatic PUFA oxidation results in products positionally the same as those catalyzed by lipoxygenases but with equal quantities of both stereoisomers $(R$ and $S$ ) at all positions of oxidation. We have recently shown that esterified, oxidized linoleate is the major oxidized PUFA in monocyte-oxidized LDL (8). Esterified, oxidized PUFA products, predominantly from linoleate, have previously been reported to be present in lesions of atherosclerosis (9-14). Kuhn et al. (14) have analyzed the oxidized PUFA products in atherosclerotic lesions using chiral phase HPLC and have recently reported detection of stereospecific oxidation in a rabbit model of the disease. Herein we report the results of our analysis of the chirality of the oxidation products in human lesions, to determine whether PUFA oxidation is catalyzed by a lipoxygenase.

The quantity of cholesteryl linoleate and its oxidation products in aortae have been shown to increase with progression of vascular disease $(9,10,13,15)$. Cholesteryl linoleate in an atherosclerotic lesion is believed to be derived from LDL that has been taken up into the lesion, since nonsteroidogenic cell membranes do not normally contain the quantities of cholesterol esters that LDL contains. The lipid content of human aortic atherosclerotic lesions is similar to that of LDL (16). Cholesteryl linoleate is the most abundant substrate for fatty acid oxidation within LDL (17). Our specific analysis of oxidized cholesteryl linoleate products thus allows examination of oxidation products putatively derived from LDL lipids within the lesions. In addition to performing a chiral analysis of oxidized

1. Abbreviations used in this paper: BHT, butylated hydroxytoluene; HODE, hydroxy-octadecadienoate; HPODE, hydroperoxy-octadecadienoate; PUFA, polyunsaturated fatty acid. 
cholesteryl linoleate in surgical specimens, the oxidation state of the oxidized cholesteryl linoleate was determined by quantitating both cholesteryl hydroperoxy-octadecadienoate (HPODE) and cholesteryl hydroxy-octadecadienoate (HODE). Cholesteryl HPODE, the primary oxidation product of cholesteryl linoleate, has not previously been specifically detected in atherosclerotic lesions.

Multiple oxidation mechanisms including oxygen or lipid radicals, peroxidative propagation, or other chemically or enzymatically catalyzed processes, likely contribute to the oxidation of LDL lipids that occurs within lesions of atherosclerosis. In addition, pure stereoisomers may isomerize to yield racemic mixtures. Detection of oxidation products with absolute stereospecificity was therefore not an expected finding. Our goal was to determine whether lipoxygenases contribute to the oxidation. To perform this evaluation, we compared the oxidized fatty acid products in human atherosclerotic lesions with those produced in vitro by copper-mediated LDL oxidation, a standard method for generating chemically oxidized LDL. The ratios of the quantities of the $S$ and $R$ stereoisomers of (ZE)-13-HODE detected in surgical samples were compared with those of copper-oxidized LDL.

\section{Methods}

Surgical sample lipid extraction. Surgical specimens of atherosclerotic lesions were obtained upon endarterectomy and included samples of lesions from carotid, aortic, femoral, or abdominal arteries. Samples of atherosclerotic plaque were also obtained from leg amputation surgeries performed on patients with vascular disease. Healthy tissue is not removed during endarterectomy, so comparisons of diseased tissue with healthy tissue obtained at endarterectomy were not possible. No longer than 10-15 min after surgical removal, the lesion specimens were dissected from the tissue and submerged in ether/ethanol (3:1) solution that also contained $500 \mu \mathrm{M}$ butylated hydroxytoluene (BHT) as an antioxidant and preservative of chiral specificity (18). The samples were promptly minced with scissors, and internal standards of cholesteryl $\left[{ }^{14} \mathrm{C}\right] \mathrm{HPODE}$, cholesteryl $\left[{ }^{14} \mathrm{C}\right] \mathrm{HODE}$, or cholesteryl $\left[{ }^{14} \mathrm{C}\right]$ linoleate (NEN Research Products, DuPont Co., Wilmington, DE) were added where indicated. The samples were allowed to extract overnight at $-20^{\circ} \mathrm{C}$. The solvent was collected, and dried under $\mathrm{N}_{2}$, and some of the samples were saponified by reconstitution in $1 \mathrm{~N} \mathrm{NaOH}$ in ethanol for $20 \mathrm{~min}$ at $60^{\circ} \mathrm{C}$. The fatty acids were purified using C18 Sep-pak columns (Millipore Corporation, Milford, MA) as previously described $(8,19)$. Samples for intact oxidized lipid analysis, to determine the oxidation state, were not saponified or processed further after drying of the extraction solvent under $\mathrm{N}_{2}$. All samples were stored at $-20^{\circ} \mathrm{C}$ under $\mathrm{N}_{2}$ until analysis.

Preparation of copper-oxidized $L D L$. LDL was isolated as previously described $(8,20,21)$. After dialysis overnight to remove EDTA added during preparation, the LDL was oxidized by incubation with 10 $\mu \mathrm{M} \mathrm{CuSO}_{4}$ in RPMI-1640 medium for $24 \mathrm{~h}$ at $37^{\circ} \mathrm{C}$, as previously described (8). At the end of the incubation, BHT ( $500 \mu \mathrm{M})$ was added to imitate the conditions of extraction for the surgical samples. The lipids were extracted (22) and saponified as previously described. The fatty acids were then purified as previously described $(8,19)$.

Oxidized fatty acid preparation for chiral phase HPLC analysis. The samples containing oxidized fatty acids from atherosclerotic plaques or $\mathrm{CuSO}_{4}$-oxidized LDL were analyzed by reversed phase HPLC as previously described $(8,23)$. The HODE positional isomers including the ${ }^{14} \mathrm{C}$-labeled HODEs derived from the internal standard were collected as they eluted from the column, BHT (and $\mathrm{NaBH}_{4}$ if necessary) was added, and the samples were immediately placed under a stream of $\mathrm{N}_{2}$ to evaporate the volatile solvents. The oxidized fatty acids were extracted from the remaining aqueous phase with an equal quantity of heptane. The heptane phase was collected and dried under $\mathrm{N}_{2}$, and the oxidized fatty acids were methylated with excess ethereal diazomethane (24).

Oxidized cholesteryl linoleate sample preparation. Some surgical specimens were extracted and the intact neutral lipids were analyzed by reversed phase HPLC as previously described (8). Ultraviolet (UV) absorbance at $206 \mathrm{~nm}$ (the absorbance of carbon-carbon double bonds) was used to detect the metabolites. Where indicated, the peaks containing cholesteryl HPODE and cholesteryl HODE were collected upon elution from the column. BHT was added, and the samples were dried under $\mathrm{N}_{2}$. The cholesteryl HPODE was reduced with $\mathrm{NaBH}_{4}$, and the cholesteryl esters were saponified. The fatty acids were extracted from the samples with heptane or purified using Sep-pak C18 columns as previously described.

Chiral phase HPLC analysis. Chiral phase HPLC was performed as described by Kuhn et al. (7) using an analytical chiral phase HPLC column with $(R)-N$-(3,5-dinitrobenzoyl)- $\alpha$-phenylglycine ionically linked over aminopropyl residues on silica gel as the chiral stationary phase (J. T. Baker Research Products, Phillipsburg, NJ). The mobile phase was $n$-hexane (Burdick \& Jackson, Muskegon, MI) with $0.5 \%$ isopropanol delivered isocratically at a flow rate of $0.7-0.8 \mathrm{ml} / \mathrm{min}$. The metabolites were monitored by UV absorbance at $236 \mathrm{~nm}$, a wavelength of light absorbed by conjugated dienes. The radioactive internal standard was detected with an on-line Flow-one Beta Radioactive Flow Detector, and Packard Radiomatic Flo-Scint I was used as scintillant (both from Radiomatic Instruments and Chemical Co., Meriden, CT). Our ability to detect stereospecific oxidation products in samples processed and analyzed by the methods described here was verified by processing and analyzing the oxidation products formed by the action of soybean lipoxygenase $\mathrm{V}$ on purified cholesteryl linoleate (both obtained from Sigma Chemical Co., St. Louis, MO; data not shown).

Data analysis. Data were collected on an IBM XT personal computer and processed using Lotus 1-2-3 (Lotus Development Corp., Cambridge, MA). Areas were determined for peaks of interest using InPlot software (GraphPAD Software, San Diego, CA), and ratios were calculated from the areas. Ratios of $S / R$ stereoisomers were compared, since this normalized the data from sample to sample. The quantity of lipid that was analyzed varied and was dependent upon the quantity of tissue extracted and the quantity of lipid in the diseased tissue. The size of individual specimens used for analysis was $\sim 1-2 \mathrm{~cm}^{2}$ and $50-300 \mathrm{mg}$ wet weight. The quantities of copper-oxidized LDL lipid that were extracted and analyzed for comparison were in the same range as the quantities of lipid present in the surgical specimens.

The Mann-Whitney two sample test was used to test all hypotheses mentioned in the text. Statistics were performed using nonparametric tests because the data did not meet criteria for parametric testing.

\section{Results}

The oxidized, esterified fatty acids from human atherosclerotic plaques and from $\mathrm{CuSO}_{4}$-oxidized $\mathrm{LDL}$ (for comparison) were analyzed to determine the relative quantities of the $S$ and $R$ stereoisomers of (ZE)-13-HODE. Shown in Fig. 1 are some representative chiral phase HPLC profiles from specimens of human atherosclerotic plaque obtained at surgery. A total of 56 samples were processed by extraction of the total lipids followed by saponification; thus, oxidized fatty acids from all lipid groups were included in the analyses. These samples included 25 specimens from carotid endarterectomy, 15 aortic specimens, 5 femoral specimens, and 11 specimens from other leg arteries. The lesion severity of the samples included 9 fatty streaks, 24 calcified lesions, 16 lesions intermediate in severity between these extremes, and 7 lesions of unknown classification. Whereas some of the specimens of atherosclerotic lesions had chiral phase HPLC profiles that showed little preference for the formation of the $S$ stereoisomer of (ZE)-13-HODE (Fig. $1 D$ ), 

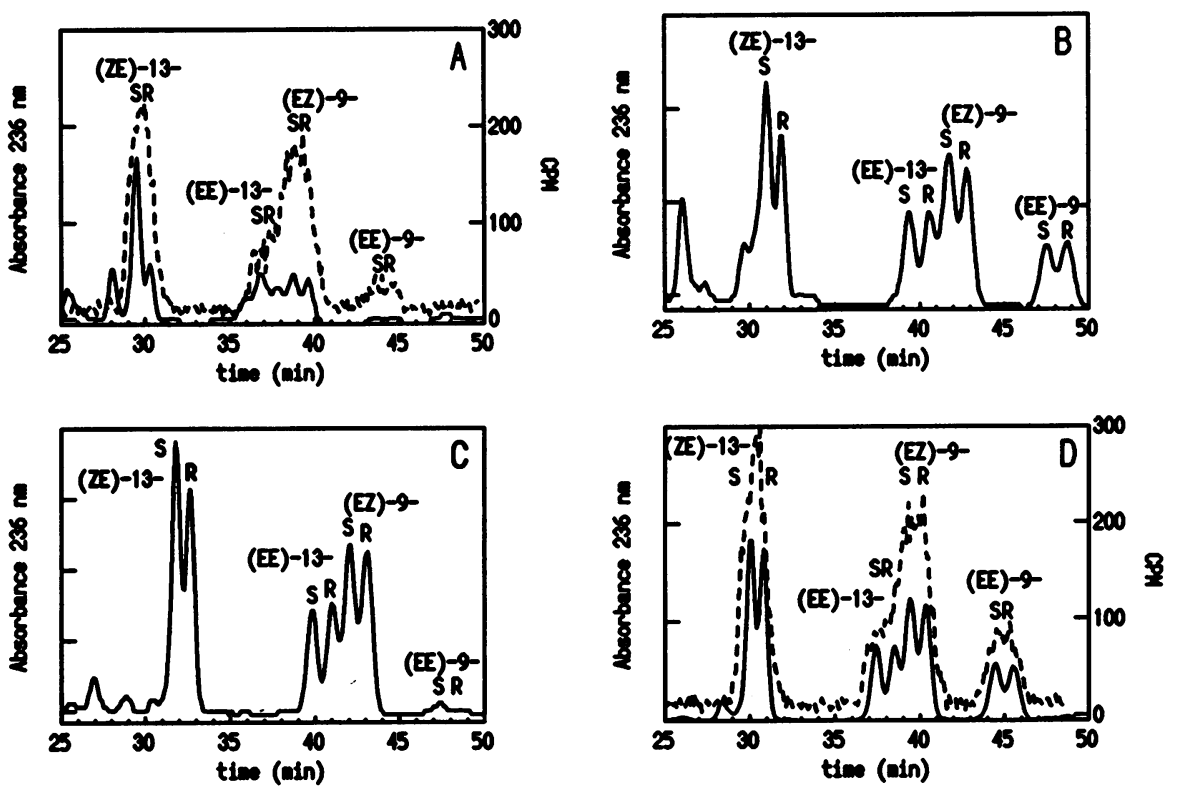

Figure 1. Stereospecifically formed oxidation products are detectable in human atherosclerotic lesions. Lipids from human atherosclerotic lesions were extracted and saponified. Racemic cholesteryl $\left[{ }^{14} \mathrm{C}\right]$ HPODE was added to some of the samples after immersing the tissue in extraction solvent. The oxidized fatty acids were separated with reversed phase HPLC, and the oxidized linoleic acid products were collected and reduced. The HODEs were methylated and then analyzed by chiral phase HPLC. The HODEs derived from the atherosclerotic lesions were detected by absorbance of conjugated dienes at $236 \mathrm{~nm}$ (solid line). The $\left[{ }^{14} \mathrm{C}\right]$ HODEs derived from the internal standard were detected by the on-line beta counter (dashed line). The pairs of stereoisomers of methylated HODE positional isomers are identified. $(A)$ Fatty streak obtained at carotid endarterectomy of an 85-yr-old male. $(B)$ Lesion from the aorta of a 71-yr-old male. $(C)$ Plaque with calcification from a carotid endarterectomy performed on a 73-yr-old male. (D) Calcified lesion obtained at carotid endarterectomy of an 85-yr-old male. other specimens processed and analyzed in exactly the same manner showed a definite difference in the quantities of $S$ and $R$ (ZE)-13-HODE ( $S>R$ ), indicating stereospecific oxidation (Fig. 1, A-C). Shown in Fig. $1 \mathrm{~A}$ (solid line) is the chiral profile of the oxidized fatty acids from a sample of fatty streak obtained at carotid endarterectomy of an 85-yr-old male. The ratio of $S / R$ stereoisomers of (ZE)-13-HODE was 3.49. Fig. 1 $B$ shows the chiral profile of a sample from the aorta of a 71yr-old male patient; the ratio of $S / R$ stereoisomers detected was 1.54. Fig. $1 C$ shows the analysis of another sample of atherosclerotic plaque from a carotid endarterectomy performed on a 73-yr-old male; this sample was characterized by some calcification. The ratio of $S / R$ stereoisomers of (ZE)-13-HODE was 1.14. The sample shown in Fig. $1 D$ was extracted from a calcified lesion obtained at carotid endarterectomy of an 85-yrold male; the $S / R$ ratio of (ZE)-13-HODE was 1.10 . The identity of peaks was verified by addition of a mixture of racemic cholesteryl $\left[{ }^{14} \mathrm{C}\right]$ HPODE to the samples (Fig. 1, $A$ and $D$, dashed lines).

In addition to verifying peak identity, the internal standard also verified that any stereospecific oxidation was not due to a step in the sample processing procedure. This radioactive internal standard was added in quantities that could be detected only with the on-line beta counter and did not interfere with the UV absorption profile of the samples. Although resolution of the $S$ and $R$ stereoisomers was not complete by radioactivity detection, a comparison of the profile of the racemic mixture of cholesteryl $\left[{ }^{14} \mathrm{C}\right]$ HPODEs in a sample without stereospecifically formed products (Fig. $1 D$ ) with that detected in a sample with stereospecifically formed products (Fig. $1 A$ ) verified that any skewing of the quantities of stereoisomers was not artifactual. The fact that some but not all of the lesion samples had some apparent stereospecificity was additional evidence that the sample processing protocol was not causing the formation of stereospecific oxidation products. Cholesteryl $\left[{ }^{14} \mathrm{C}\right]$ linoleate was also added to some of the samples to detect any lipid oxidation that might be occurring during the sample extraction and processing. No $\left[{ }^{14} \mathrm{C}\right]$ HODEs were detected in samples spiked with cholesteryl $\left[{ }^{14} \mathrm{C}\right]$ linoleate (data not shown). The limit of detection using the online $\beta$-counter for $\left[{ }^{14} \mathrm{C}\right] \mathrm{HODEs}$ was $\sim 0.5 \mathrm{pmol}$; the quantities of HODEs detected in surgical specimens by UV absorbance were in the nanomolar range.

The mean $S / R$ ratios determined for (ZE)-13-HODE obtained from atherosclerotic plaque were all significantly different from 1.00 (Table I). Because the ratios of $S / R$ stereoisomers of (ZE)-13-HODE varied among surgical samples, it was also necessary to determine how much the ratios varied in nonenzymatic oxidation. To accomplish this, samples of $\mathrm{CuSO}_{4}$-oxidized LDL were extracted, processed, and analyzed just as the lesion lipids had been (Fig. 2). The areas under the $S$ and $R$ peaks of (ZE)-13-HODE found with $\mathrm{CuSO}_{4}$-mediated $\mathrm{LDL}$ oxidation were quantitated and analyzed, and the ratio of $S / R$ stereoisomers was found to be significantly less than the ratio of 13- $(S)$ - to 13- $(R)$-HODE detected from specimens of atherosclerotic lesion (Table I).

LDL is the major source of the lipid that accumulates in atherosclerotic lesions $(1-4,16)$, and cholesteryl linoleate is the most abundant substrate for oxidation within LDL (17). Cholesteryl linoleate content of atherosclerotic aortae has specifically been found to increase with severity of disease (15), and linoleate is the major PUFA esterified to cholesterol in atherosclerotic lesions (25). For these reasons, we separately analyzed the fatty acid oxidation products obtained from cholesteryl linoleate. The intact lipids from surgical samples were analyzed using a reversed phase HPLC method that resolves neutral lipids (reference 8; Fig. 3). In the top panel of Fig. 3, a representative sample of a profile of neutral lipids extracted from an atherosclerotic lesion obtained at endarterectomy of a 65-yr-old female is shown. Peak 1 contains free cholesterol, peak 2 contains chölesteryl HPODE, peak 3 contains cholesteryl HODE, and peak 4 contains cholesteryl linoleate. The extinction coefficients for calculating cholesterol con- 


\begin{tabular}{|c|c|c|c|}
\hline & \multicolumn{2}{|c|}{$S / R$ ratio } & \multirow[b]{2}{*}{ Plaque versus $\mathrm{CuSO}_{4}$} \\
\hline & Plaque & $\mathrm{CuSO}_{4}$ & \\
\hline Total extracted lipids & $1.12 \pm 0.045 *(1.08) n=56$ & $1.03 \pm 0.010 \quad(1.02) n=30$ & $P=0.0004$ (one-tailed) \\
\hline Cholesteryl HPODE & $\begin{array}{l}1.49 \pm 0.159^{\ddagger}(1.22) n=18 \\
\text { (females: } 1.58 \pm 0.28, n=9)^{\| * *} \\
\text { (males: } 1.37 \pm 0.15, n=9)^{1 * *}\end{array}$ & $1.03 \pm 0.015^{8 \| 9}(1.06) n=13$ & $P=0.0002$ (one-tailed) \\
\hline Cholesteryl HODE & $1.20 \pm 0.107^{\ddagger}(1.09) n=18$ & $0.98 \pm 0.040^{8} \quad$ (1.03) $n=8$ & $P=0.0085$ (one-tailed) \\
\hline
\end{tabular}

The lipids were extracted from samples of human atherosclerotic plaque or $\mathrm{CuSO}_{4}$-oxidized LDL, and the oxidized lipids were further purified by reversed phase HPLC. Chiral phase HPLC analysis was used to determine the ratio of $S / R$ stereoisomers of (ZE)-13-HODE from the total oxidized, esterified fatty acids or (ZE)-13-HODE from the purified cholesteryl HPODE or cholesteryl HODE. The data are expressed as the mean \pm SE, and the median is shown in parentheses. All plaque ratios were significantly different from 1.00. * Cholesteryl HPODE versus total extracted lipids from plaque: two-tailed $P=0.0003$. ${ }^{\ddagger}$ Cholesteryl HPODE versus cholesteryl HODE from plaque: two-tailed $P=0.0144$. ${ }^{\S} \mathrm{Cholesteryl} \mathrm{HPODE}$ versus cholesteryl HODE from $\mathrm{CuSO}_{4}$ : two-tailed $P=0.3284$. "Cholesteryl HPODE from females versus cholesteryl $\mathrm{HPODE}$ from CuSO ${ }_{4}$ : onetailed $P<0.0001$. ' Cholesteryl HPODE from males versus cholesteryl HPODE from $\mathrm{CuSO}_{4}$ : one-tailed $P=0.0082$. ** Cholesteryl HPODE from females versus cholesteryl HPODE from males: two-tailed $P=0.2895$.

tent in the labeled peaks were previously determined (8) and allowed quantitation of cholesteryl HPODE, cholesteryl HODE, and cholesteryl linoleate in the atherosclerotic lesions. From these samples, the oxidation state of the oxidized cholesteryl linoleate was determined as well as the extent of oxidation of available substrate within the lesions. Cholesteryl $\left[{ }^{14} \mathrm{C}\right]$ HPODE added to the samples as an internal standard verified that reduction was not occurring during sample processing (data not shown). The quantities of the primary oxidation products, cholesteryl HPODE and cholesteryl HODE, were determined for 19 specimens. Overall, the samples contained $42 \%$ of the cholesteryl linoleate as cholesteryl HPODE ( $58 \pm 12 \%$ cholesteryl HODE). The presence of the hydroxy-form of oxidized cholesteryl linoleate suggests a reduction mechanism in the atherosclerotic lesion.
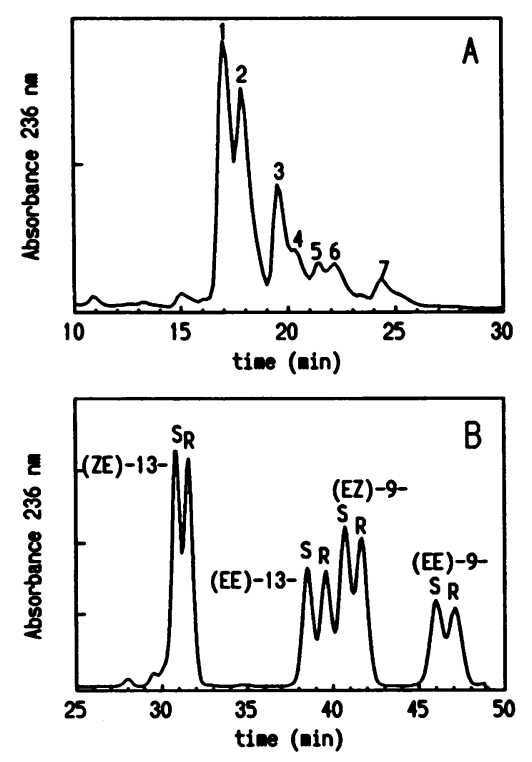

Figure 2. Chiral phase HPLC analysis of $\mathrm{CuSO}_{4}$-oxidized LDL. Human LDL was isolated and oxidized by exposure to $\mathrm{CuSO}_{4}$ as described in Methods. The lipids were extracted and saponified, and the oxidized fatty acids were resolved by reversed phase HPLC $(A)$. The oxidized, esterified linoleic acid products were collected, reduced, methylated, and analyzed by chiral phase HPLC $(B)$. In $B$, the racemic pairs of stereoisomers of the positional isomers of methylated HODEs are labeled. In $A$, peak 1 is (ZE)-13-HODE; peak 2,

(EZ)-9-HODE; peak 3, (EE)-13- and 9-HODE; peak 4, (ZE)-13HPODE; peak 5, (EZ)-9-HPODE; peak 6, (EE)-13-HPODE; and peak 7, (EE)-9-HPODE.
The total quantities of cholesteryl linoleate and its primary oxidation products ranged from 0.5 to $1.0 \mu \mathrm{mol}$ per sample. The extent of oxidation was calculated by dividing the quantity of cholesteryl HPODE and cholesteryl HODE by the quantity of cholesteryl HPODE, cholesteryl HODE, and cholesteryl linoleate (8). This calculation yielded a value for percent oxidation of available cholesteryl linoleate substrate. The percentage of available substrate detected as primary oxidation products ranged from 1 to $13 \%$. There did not appear to be any correlation between the quantity (percentage) of primary oxidation products and the stage of lesion severity as determined by gross examination (data not shown).

For some samples, the peaks containing cholesteryl HPODE and cholesteryl HODE were collected separately and further

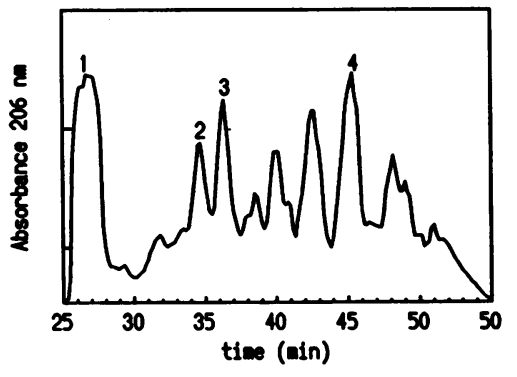

Figure 3. Chiral phase HPLC analysis of cholesteryl HPODE and cholesteryl HODE purified from an atherosclerotic lesion. The lipids were extracted from an atherosclerotic lesion obtained at endarterectomy performed on a 65 -yr-old female patient. The neutral lipids were resolved by reversed phase HPLC (upper chromatogram). Peak $l$ contains free cholesterol; peak 2, cholesteryl HPODE; peak 3, cholesteryl HODE; peak 4 , cholesteryl linoleate. Peaks 2 and 3 were collected, reduced, saponified, methylated, and analyzed by chiral phase HPLC. The chiral phase

HPLC profiles of the methylated (ZE)-13-HODE obtained from the cholesteryl HPODE collected in peak 2 (bottom left) and from the cholesteryl HODE collected in peak 3 (bottom right) are shown. 
processed by reduction and saponification for chiral phase HPLC analysis. Included in the analyses of cholesteryl HPODE were 11 carotid endarterectomy specimens, 5 aortic specimens, and 2 femoral specimens. Among these specimens, 11 were calcified, 3 were intermediate in severity (between fatty streak and calcified), and 4 were of unknown severity. The samples for cholesteryl HODE analysis included 12 carotid endarterectomy specimens, 4 aortic specimens, and 2 femoral specimens. Among these, 10 were calcified, 2 were intermediate in severity, and 6 were of unknown severity. Whereas some samples contained sufficient oxidation products for both cholesteryl HPODE and cholesteryl HODE to be analyzed, other samples had only enough material for chiral phase HPLC analysis of one of the oxidation states.

A representative sample is shown in Fig. 3. The ratio of $S / R$ stereoisomers for the (ZE)-13-HODE derived from cholesteryl HPODE was 1.21 (Fig. 3, peak 2), and the $S / R$ ratio for the (ZE)-13-HODE derived from cholesteryl HODE was 1.16 (Fig. 3 , peak 3 ). The (ZE)-13-HODE $S / R$ ratios were determined for all of the samples processed in this manner. The mean ratio was higher in the cholesteryl HPODE than the cholesteryl HODE extracted from atherosclerotic lesions (Table I). The (ZE)-13-HODE $S / R$ ratios from the cholesteryl HPODE products purified from atherosclerotic lesions were also noticeably higher than the (ZE)-13-HODE $S / R$ ratios from surgical samples for which the total oxidized fatty acids were analyzed (Table I). When the $S / R$ ratio of the (ZE)-13-HODE from cholesteryl HPODE, extracted from atherosclerotic lesions, was compared with the same purified oxidation product from $\mathrm{CuSO}_{4}$-oxidized LDL extracted and analyzed in the same manner, the differences were significant (Table I). There was no significant difference in the $S / R$ ratio of (ZE)-13-HODE derived from cholesteryl HPODE and cholesteryl HODE purified from $\mathrm{CuSO}_{4}$-oxidized LDL ( Table I). In addition, we separately compared the $S / R$ ratios from the cholesteryl HPODE-derived (ZE)-13-HODE from male and female patients with the same oxidation product derived from $\mathrm{CuSO}_{4}$-oxidized LDL. The stereospecificity of this oxidation product was significantly greater than that from $\mathrm{CuSO}_{4}$-oxidized LDL in both genders (Table I). There was no significant difference in stereospecificity of oxidation between males and females (Table I).

Lesions of vascular disease have been described as progressing through stages, first visible grossly as the fatty streak and increasing in size with increasing cholesterol deposition. Lesions progressively become calcified, and in the most severe cases ulcerations appear. (26) For comparison of stereospecificity of oxidation in different stages of lesion formation, three samples were dissected from each patient's tissue, including a sample of fatty streak, a section of more advanced, calcified lesion, and a sample of lesion intermediate in severity between these two extremes. These samples came from five patients undergoing carotid endarterectomy and two patients undergoing leg amputation. The total oxidized, esterified fatty acids were extracted, saponified, purified, and analyzed by chiral phase HPLC. Among samples analyzed in this manner, there were no general trends in stereospecificity of oxidation detected with differing stages of lesion formation (data not shown).

\section{Discussion}

While considerable evidence exists for the presence of oxidized lipids and oxidized LDL in atherosclerotic lesions (1-5), no mechanism for lipid oxidation has been definitively demonstrated to occur in human lesions. A role for the enzyme 15lipoxygenase has generated considerable interest, since it is present in lesions in the same location as macrophages and oxidized LDL epitopes (5). Our laboratory has previously shown that purified 15-lipoxygenase from soybeans can oxidize LDL and render it toxic to proliferating fibroblasts (27). Purified 15-lipoxygenase has also been shown to be capable of oxidizing the esterified fatty acids in LDL with partial stereospecificity $(28,29)$. It is the stereospecific nature of 15 -lipoxygenase oxygenation that allows specific detection of 15-lipoxygenase activity in samples containing oxidized fatty acids (6, $7)$. The stereospecificity of only the 13 positional isomer ( $(\mathrm{ZE})$-13-HODE in reduced samples) was examined because this product has been shown to be the positional isomer that is stereospecifically formed (13-( $S)$-HPODE) by 15-lipoxygenase $(12,28) .9-\mathrm{HPODE}$ and the EE-isomers of both 13- and 9-HPODE are minor products or the products of isomerization of 13-HPODE and lack stereospecificity $(7,12,28)$.

We have examined the oxidized fatty acids in human atherosclerotic lesions for evidence of lipoxygenase activity in vivo. Kuhn et al. (14) have recently found evidence of 15-lipoxygenase activity in vivo in atherosclerotic lesions induced in rabbits by cholesterol feeding. They reported that detection of 15-lipoxygenase activity correlated with cholesterol deposition in rabbit arteries. In our extensive analysis of human atherosclerotic lesions, we have obtained results that corroborate the animal model studies of Kuhn et al. (14). We have detected stereospecifically formed, oxidized, esterified linoleate in samples of human atherosclerotic plaque. In contrast, Kuhn et al. (14) reported negative results regarding detection of stereospecific oxidation in advanced human atherosclerotic plaques. Differences in sample processing protocols or patient disease status at the time of surgery may account for the differing results. The size of tissue samples analyzed differed: our samples were $\sim 50-300 \mathrm{mg}$ wet weight and contained up to 20 times less tissue than those of Kuhn et al. (14). More tissue might result in a dilution of the plaque, LDL-derived, oxidized lipids by oxidized lipids from the surrounding tissue. Furthermore, our detection of greater stereospecificity in cholesteryl linoleate oxidation products than in the total, esterified, oxidized linoleate would suggest that dilution of the stereospecifically formed oxidation products by nonstereospecifically formed oxidation products was occurring in total lipid extracts. The limited sample size may also have been responsible for observed differences, since in the previously reported analysis only 10 human surgical specimens were examined (14).

Our finding of a few samples with particularly pronounced differences in the $S / R$ ratio of (ZE)-13-HODE can be explained in several ways. Factors such as the phase of lesion development, the type of disease induction, or individual variation may affect the mechanisms that cause oxidation of lipids to occur in lesions. One possibility is that these lesions were undergoing a period of enzymatic oxidation similar to that which occurs during cholesterol deposition in the rabbit model of atherosclerosis described by Kuhn et al. (14). Another possible explanation is that enzyme-mediated oxidation contributes to a greater extent in some individuals than in others. This could be due to environmental factors such as dietary differences or smoking, or genetic differences among individuals. Our detection of a predominance of the $S$ stereoisomer in some, but not all, of the lesions also indicates that some instability intrinsic to the $R$ 
stereoisomer is not causing the skewing. If the $R$ stereoisomer were less stable than the $S$ stereoisomer, (ZE) 13-S-HODE would predominate in all lesions analyzed.

We have separately analyzed the primary oxidation products derived from cholesteryl linoleate in human atherosclerotic lesions. These metabolites were of particular interest because they are the primary oxidation products formed from substrate likely derived from LDL lipid. While cholesteryl HPODEs have not previously been specifically detected and quantitated, their presence in atherosclerotic lesions has been suggested (11). The cholesteryl linoleate content of aortae has been reported to increase with progression of atherosclerotic disease (15), potentially providing more substrate for oxidation. It is interesting that we have detected greater stereospecificity of oxidation among the primary oxidation products from a substrate that becomes deposited in the vessel wall as the disease progresses. Peroxidized lipids have also been reported to increase in quantity with increasing pathological change $(9,10,13,30)$. YlaHerttuala et al. (5) have shown that 15-lipoxygenase mRNA and protein are present where macrophages and oxidized LDL are present. Although the order of events in the development of the disease is not certain, our results suggest that lipoxygenase activity plays a role in the initiation of oxidation, which may then propagate nonenzymatically.

Initiation of oxidation may not consist of a single event in the progression of lesion formation. In all lesions analyzed using the reversed phase HPLC method that separates neutral lipids, cholesteryl HPODE was detected, regardless of the stage of lesion progression. The fact that primary oxidation products, and not just breakdown products, are found in all lesions suggests that initial product formation is ongoing. This idea is supported by the fact that 15-lipoxygenase protein and mRNA were previously detected in all stages of disease (5). We additionally have not found any correlation between detection of stereospecific oxidation and the stage of the lesion analyzed. An enzymatic oxidation mechanism does not necessarily have to occur during a stage that has been chosen for convenient classification of lesions by gross examination. There may be some low level of enzyme-mediated oxidation in all stages of atherosclerotic disease, which in response to external events (e.g., diet, heredity, lipid deposition) may become more pronounced. Additionally, our results may indicate that the primary oxidation products are relatively stable in the environment of the lesion.

We have also estimated the extent of oxidation of cholesteryl linoleate in the specimens and found that $1-13 \%$ of the cholesteryl linoleate was detected as the primary oxidation products (cholesteryl HPODE or cholesteryl HODE). Our determination is an underestimate of total oxidation because we analyzed only the primary oxidation products. Kuhn et al. (13) have previously made the same determination including cholesteryl HODE and cholesteryl keto-octadecadienoate and found a range of oxidation of cholesteryl linoleate from 12.4 to $21 \%$. Although previous determinations have found a correlation between total oxidized lipid content and the stage of disease $(9,10,13,30)$, our analysis of primary oxidation products did not reveal such a correlation. Thus, although the total content of oxidation products may increase with disease progression, the rate of primary product formation and breakdown may vary throughout progression of the disease.

Herein we report the first specific detection of cholesteryl HPODE in atherosclerotic lesions. We have also detected the reduction product, cholesteryl HODE. The presence of the reduced form in lesions suggests a reduction mechanism. One possible enzymatic reduction mechanism is the phospholipid hydroperoxide glutathione peroxidase that reportedly acts on cholesteryl ester hydroperoxide substrate (31). Peroxidase activity has been detected in atherosclerotic lesions of pigeons (32). Myeloperoxidase, an enzyme with peroxidase activity that also has the capacity to generate hypochlorous acid, an oxidizing agent, has been detected in human atherosclerotic lesions (33). Another possible mechanism may be the peroxidase activity that has been reported for lipoxygenase (34). We have analyzed the $S / R$ ratio of (ZE)-13-HODE derived from both cholesteryl HPODE and cholesteryl HODE in order to determine whether there was a difference in the ratios from these two oxidation states. Our finding of a higher $S / R$ ratio in the (ZE)-13-HODE derived from cholesteryl HPODE as compared with that derived from cholesteryl HODE rules out the possibility that a peroxidase-type activity or any other reduction mechanism is responsible for the formation of stereospecific products.

The process by which 15-lipoxygenase contributes to the formation of oxidized lipids in atherosclerotic lesions is unclear. 15-Lipoxygenase is believed to be an intracellular enzyme. Our laboratory has previously shown that oxidation of LDL by human monocytes appears to involve a lipoxygenase, and a role for the enzyme in regulating monocyte activation and related LDL oxidation was previously proposed $(35,36)$. It is possible that the apparent requirement for lipoxygenase activity in monocyte oxidation of LDL is a process distinct from the lipoxygenase-mediated oxidation of lipids in atherosclerotic plaques. Questions remain regarding the cellular location of 15-lipoxygenase and LDL when it becomes oxidized. There are several possible scenarios for enzymatic oxidation of lipids derived from LDL in atherosclerotic lesions. Intracellular 15-lipoxygenase may oxidize cellular lipids that are then transferred to LDL and propagate the oxidation of LDL lipids. It is also possible that cell injury results in release of 15-lipoxygenase to the extracellular environment, where it contacts LDL. Alternatively, modified LDL may be taken up by foam cells and then modified further by intracellular 15-lipoxygenase. Future studies will reveal the mechanisms involved.

We have determined that stereospecific oxidation, consistent with lipoxygenase activity, is detectable in human lesions of atherosclerotic. We have presented data supporting the concept that enzymatic lipid oxidation occurs during lesion development in human peripheral vascular disease. Our results do not allow us to distinguish between 15- and 12-lipoxygenase activity, because both have been reported to stereospecifically catalyze the oxidation of esterified linoleate (29). However, nor do they rule out another protein with lipoxygenase activity. 15-Lipoxygenase has previously been shown to be present where macrophages and oxidized LDL are located in human atherosclerotic lesions (5). The activity is more apparent when oxidation products specifically derived from LDL lipids are analyzed (Table I). Kuhn et al. (14) have detected similar enzymatic activity in a rabbit model of atherosclerosis and found it to coincide with cholesterol deposition in the arterial wall. Together these results support the current speculation that 15-lipoxygenasemediated oxidation represents an attempt by macrophages to rid the atherosclerotic vessel wall of excess lipid by metabolizing it and preparing it for further breakdown, just as reticulocytes use 15-lipoxygenase in catabolizing membranes of mitochondria. 
Inhibition of this process may provide a means for impeding the progression of atherosclerotic disease.

\section{Acknowledgments}

We wish to gratefully acknowledge the help of Mary Posey in coordinating the collection of surgical specimens. We also thank Dr. Guy M. Chisolm for reviewing the manuscript.

This work was funded in part by a fellowship grant from the American Heart Association, Northeast Ohio Affiliate (F316; V. A. Folcik), a National Research Service Award from the National Heart, Lung, and Blood Institute, NIH (1 F32 HL08904-01; V. A. Folcik), and grants from the National Institutes of Health (HL 51068; M. K. Cathcart) and the National American Heart Association (91-1201; M. K. Cathcart).

\section{References}

1. Penn, M. S., and G. M. Chisolm. 1994. Oxidized lipoproteins, altered cell function and atherosclerosis. Atherosclerosis. 108(Suppl.):S21-S29.

2. Chisolm, G. M., and M. S. Penn. 1996. Oxidized lipoproteins and atherosclerosis. In Atherosclerosis and Coronary Disease. V. Fuster, R. Ross, and E. Topol, editors. Raven Press, New York. 129-149.

3. Palinski, W., M. E. Rosenfeld, S. Yla-Herttuala, G. C. Gurtner, S. S. Socher, S. W. Butler, S. Parthasarathy, T. E. Carew, and D. Steinberg. 1989. Low density lipoprotein undergoes oxidative modification in vivo. Proc. Natl. Acad. Sci. USA. 86:1372-1376

4. Yla-Herttuala, S., W. Palinski, M. E. Rosenfeld, S. Parthasarathy, T. E. Carew, S. Butler, J. L. Witzum, and D. Steinberg. 1989. Evidence for the presence of oxidatively modified low density lipoprotein in atherosclerotic lesions of rabbit and man. J. Clin. Invest. 84:1086-1095.

5. Yla-Herttuala, S., M. E. Rosenfeld, S. Parthasarathy, E. Sigal, T. Sarkioja, J. L. Witzum, and D. Steinberg. 1991. Gene expression in macrophage-rich human atherosclerotic lesions. 15-Lipoxygenase and acetyl low density lipoprotein receptor messenger RNA colocalize with oxidation specific lipid-protein adducts. $J$. Clin. Invest. 87:1146-1152.

6. Hamberg, M., and B. Samuelsson. 1967. On the specificity of the oxygenation of unsaturated fatty acids catalyzed by soybean lipoxidase. J. Biol. Chem. 242:5329-5335

7. Kuhn, H., R. Wiesner, V. Z. Lankin, A. Nekrasov, L. Adler, and T. Schewe. 1987. Analysis of the stereochemistry of lipoxygenase-derived hydroxypolyenoic fatty acids by means of chiral phase high-pressure liquid chromatography. Anal. Biochem. 160:24-34.

8. Folcik, V. A., and M. K. Cathcart. 1994. Predominance of esterified hydroperoxy-linoleic acid in human monocyte-oxidized LDL. J. Lipid Res. 35:15701582.

9. Harland, J. D., J. D. Gilbert, G. Steel, and C. J. W. Brooks. 1971. Lipids of the human atheroma. Part 5. The occurrence of a new group of polar sterol esters in various stages of human atherosclerosis. Atherosclerosis. 13.239-246.

10. Belkner, J., R. Wiesner, and H. Kuhn. 1992. Identification of oxidatively modified lipids in atherosclerotic lesions of human aortas. Agents Actions. Suppl. 37:78-84.

11. Brooks, C. J. W., G. Steel, J. D. Gilbert, and W. A. Harland. 1971. Lipids of the human atheroma. Part 4. Characterization of a new group of polar sterol esters from human atherosclerotic plaques. Atherosclerosis. 13:223-237.

12. Belkner, J., R. Weisner, H. Kuhn, and V. Z. Lankin. 1991. The oxygenation of cholesterol esters by the reticulocyte lipoxygenase. FEBS (Fed. Eur. Biochem. Soc.) Lett. 279:110-114.

13. Kuhn, H., J. Belkner, R. Wiesner, T. Schewe, V. Z. Lankin, and A. K. Tikhaze. 1992. Structure elucidation of oxygenated lipids in human atherosclerotic lesions. Eicosanoids. 5:17-22.
14. Kuhn, H., J. Belkner, S. Zaiss, T. Fahrenklemper, and S. Wohlfeil. 1994. Involvement of 15-lipoxygenase in early stages of atherogenesis. J. Exp. Med. 179:1903-1911.

15. Bottcher, C. J. F., F. P. Woodford, C. Ch. T. H. Romeny-Wachter, E. B. Van Houte, and C. M. Van Gent. 1960. Fatty acid distribution in lipids of the aortic wall. Lancet. 1378-1383.

16. Mukhin, D. N., A. N. Orekhov, E. R. Andreeva, E. M. Schindeler, and V. N. Smirnov. 1991. Lipids in cells of atherosclerotic and uninvolved human aorta. III. Lipid distribution in intimal sublayers. Exp. Mol. Pathol. 54:22-30.

17.-Scanu, A. M. 1979. Plasma lipoproteins: an introduction. In The Biochemistry of Atherosclerosis. A. M. Scanu, editor. Marcel Dekker, Inc., New York. $3-8$

18. Kuhn, H., J. Belkner, R. Wiesner, and A. R. Brash. 1990. Oxygenation of biological membranes by the pure reticulocyte lipoxygenase. J. Biol. Chem. 265:18351-18361.

19. Powell, W. S. 1982. Rapid extraction of arachidonic acid metabolites from biological samples using octadecylsilyl silica. Methods Enzymol. 86:467-477.

20. Havel, R. J., H. A. Eder, and J. H. Bragdon. 1955. The distribution and chemical composition of ultracentrifugally separated lipoproteins in human serum. J. Clin. Invest. 34:1345-1353.

21. Hatch, F. T., and R. S. Lees. 1968. Practical methods for plasma lipoprotein analysis. Adv. Lipid Res. 6:1-68.

22. Bligh, E. G., and W. J. Dyer. 1959. A rapid method of total lipid extraction and purification. Can. J. Biochem. Physiol. 37:911-917.

23. Claeys, M., G. A. A. Kivits, E. Christ-Hazelhof, and D. H. Nugteren. 1985. Metabolic profile of linoleic acid in porcine leukocytes through the lipoxygenase pathway. Biochim. Biophys. Acta. 837:35-51.

24. Schlenk, H., and J. C. Gellerman. 1960. Esterification of fatty acids with diazomethane on a small scale. Anal. Chem. 32:1412-1414.

25. Rapp, J. H., W. E. Connor, D. S. Lin, T. Inahara, and J. M. Porter. 1983. Lipids of human atherosclerotic plaques and xanthomas: clues to the mechanism of plaque progression. J. Lipid Res. 24:1329-1335.

26. Ross, R. 1988. The pathogenesis of atherosclerosis. In Heart Disease: A Textbook of Cardiovascular Medicine. 3rd ed. Eugene Braunwald, editor. W. B. Saunders Co., Philadelphia, PA.

27. Cathcart, M. K., A. K. McNally, and G. M. Chisolm. 1991. Lipoxygenasemediated transformation of human low density lipoprotein to an oxidized and cytotoxic complex. J. Lipid Res. 32:63-70.

28. Belkner, J., R. Wiesner, J. Rathman, J. Barnett, E. Sigal, and H. Kuhn. 1993. Oxygenation of lipoproteins by mammalian lipoxygenases. Eur. J. Biochem. 213:251-261

29. Kuhn, H., J. Belkner, H. Suzuki, and S. Yamamoto. 1994. Oxidative modification of human lipoproteins by lipoxygenases of different positional specificities. J. Lipid Res. 35:1749-1759.

30. Glavind, J., S. Hartmann, J. Clemmesen, K. E. Jessen, and H. Dam. 1952. Studies on the role of lipoperoxides in human pathology. II. The presence of peroxidized lipids in the atherosclerotic aorta. Acta Pathol. Microbiol. Scand. 30:1-6.

31. Thomas, J. P., P. G. Geiger, M. Maiorino, F. Ursini, and A. W. Girotti. 1990. Enzymatic reduction of phospholipid and cholesterol hydroperoxides in artificial bilayers and lipoproteins. Biochim. Biophys. Acta. 1045:252-260.

32. Taylor, R. G., W. G. Jerome, and J. C. Lewis. 1992. Ultrastructural localization of peroxidase in atherosclerotic lesions of pigeons. Exp. Mol. Pathol. 57:167-179.

33. Daugherty, A., J. L. Dunn, D. L. Rateri, and J. W. Heinecke. 1994. Myeloperoxidase, a catalyst for lipoprotein oxidation, is expressed in human atherosclerotic lesions. J. Clin. Invest. 94:437-444.

34. Hartel, B., P. Ludwig, T. Schewe, and S. M. Rapoport. 1982. Self-inactivation by 13-hydroperoxylinoleic acid and lipohydroperoxidase activity of the reticulocyte lipoxygenase. Eur. J. Biochem. 126:353-357.

35. McNally, A. K., G. M. Chisolm, D. W. Morel, and M. K. Cathcart. 1990. Activated human monocytes oxidize LDL by a lipoxygenase dependent pathway. J. Immunol. 145:254-259.

36. Folcik, V. A., and M. K. Cathcart. 1993. Assessment of 5-lipoxygenase involvement in human monocyte-mediated LDL oxidation. J. Lipid Res. 34:6979. 